\title{
A Method Based on Fiber Raman Scattering Cable for Testing Temperature and Reducing Noise
}

\author{
Chunbo LIU1', Liangyi Huang, Rui LI, Zhidong GU and Jiawei XU \\ Information and Communications Branch of Hainan Power Grid Co. Ltd, Haikou 570100, China \\ 1878512868@qq.com
}

Keywords: Wavelet transform. Empirical mode analysis. Noise reduction.

Abstract.According to the characteristics of Raman fiber signal, combined with the advantages of wavelet threshold denoising and empirical mode method, the two methods are combined for removing noise. This method improves the signal-to-noise ratio by $15 \%$ in simulation experiments. This will get more accurate cable temperature data in practical application.

\section{Introduction}

According to the principle of Raman fiber temperature,the intensity of backscattering Raman scattering is 10 negative 9 times of the incident light intensity, while the anti Stokes light and Stokes light are a few orders of magnitude. And the output fluctuation of the pulse laser, the optical fiber bending loss, the uneven light material and the noise produced in the light transmission have great influence on the signal.In most optical fiber sensing systems, optical signals are often converted into electrical signals, which will produce a lot of noise.Therefore, when using optical fiber to measure the temperature of the cable, the temperature signal is very weak compared with the noise signal.The signal denoising is of great significance to obtain the accurate of cable temperature.

\section{The research status of noise reduction method for optical fiber temperature measurement data}

The original signal of Raman fiber has low signal-to-noise ratio and its noise is mainly white noise and random noise; the useful signal is low frequency signal, and noise is high frequency signal.At present, the commonly used methods in optical fiber signal processing are cumulative averaging algorithm and wavelet transform method.The signal-to-noise ratio (SNR) of cumulative averaging algorithm increases with the increase of cumulative number. However, the signal-to-noise ratio (SNR) is not significantly improved after increasing to a certain number.Wavelet analysis has multi-scale characteristics. It can observe signals at a variety of resolutions and make a good representation of the local characteristics of the signal in time domain and frequency domain,effectively remove the noise in the useful signal.And the application tends to mature at present $^{[1,2]}$.

\section{Wavelet analysis}

Set the signal to be measured

$$
f(t)=s(t)+n(t) \quad t=0,1,2, \ldots, N-1
$$


In, $\mathrm{s}(\mathrm{t})$ is useful signal;n(t) is obedience to the Gauss distribution and variance is the sigma 2 of noise signal.The wavelet transform transforms the noisy signal from the time domain to the frequency domain, and removes the noise in the signal by the frequency domain characteristic of the useful signal and noise.First,according to the signal characteristics select the appropriate wavelet function to decompose the signal and get a set of wavelet coefficients. Then, a suitable threshold is selected to process the decomposed signal.Finally, the signal is obtained through wavelet reconstruction after denoising ${ }^{[3]}$.

\section{The de-noising method of empirical mode combined with wavelet analysis}

The empirical mode analysis method separates a number of intrinsic eigenfunctions from the oscillations of the original time scale step by step.The expression of empirical mode decomposition is:

$$
f(t)=\sum_{i=1}^{n-1} m_{i}(t)+h_{n}(t)
$$

The original noise - containing signal $f(t)$ is decomposed into $n-1$ signal sequences with different scales of $m_{i}(t)$ and a residual term $h_{n}(t)$ through the hierarchical screening process, and the terminating condition is the Preset threshold value:

$$
S D=\sum_{t=0}^{T}\left[\frac{\left|h_{n-1}(t)-h_{n}(t)\right|^{2}}{h_{n-1}^{2}(t)}\right]
$$

The selection of terminating conditions is very important. When oversize will lead to the IMF component can not meet the requirements,and the small IMF component will be become a frequency modulated signal with constant amplitude. In general, SD usually is $0.2-0.3^{[4]}$.

\section{The de-noising method of empirical mode combined with wavelet analysis}

The steps of de-noising method combined with empirical mode analysis and wavelet analysis are as follows:

(1) The original signal is decomposed.The IMF component and a residual quantity are obtained.

(2) Threshold processing for the former $r$ components to get a new IMF component.

(3) Using the IMF component after de-noising and residual component to reconstruct the signal, the new signal can be expressed as:

$$
\hat{f}(t)=\sum_{1}^{r} \hat{m}_{i}(t)+\sum_{r+1}^{n-1} m_{i}(t)+\hat{h}_{n}(t)
$$

$\hat{f}(t)$ is denoising signal. $\hat{m}_{i} 、 \hat{h}_{n}$ is components after wavelet denoising.

The advantage of this method is that it can retain some useful signals in the high-frequency IMF component after EMD decomposition, and do not need to select the basis functions.

\section{The simulation experiment of signal de-noising}

According to the above theory, wavelet denoising and empirical mode combined with wavelet denoising are carried out in this paper. Wavelet denoising selects db4 wavelets with good smoothness and strong localization in frequency domain as wavelet bases. The number of 
decomposition layers is 5 , and the threshold function is soft threshold function.Empirical mode combined with wavelet denoising method decomposes the original signal by EMD,then reconstructs the signal after Threshold de-noising for the former $\mathrm{r}$ components.

The root mean square error and SNR of the two methods are compared as follows:

table 3_1 The root mean square error and signal-to-noise ratio of two methods after denoising

\begin{tabular}{|c|c|c|}
\hline de-noising method & wavelet de-noising & $\begin{array}{c}\text { empirical mode combined } \\
\text { with wavelet analysis }\end{array}$ \\
\hline signal-to-noise ratio & 11.5427 & 13.2709 \\
\hline Root mean square error & 0.2036 & 0.1843 \\
\hline
\end{tabular}

\section{Conclusions}

The de-noising method of empirical mode combined with wavelet analysis can effectively improve signal to noise ratio and reduce root mean square error.It can meet the requirement of signal processing in temperature measurement system.At the same time,this method avoids the choice of wavelet base and decomposition level, and can effectively avoid the wrong choice of wavelet function and decomposition level caused by lack of experience.

\section{References}

[1] SunBaiNing, Noise analysis and optimization of distributed Raman fiber optic temperature sensing system[D].Shandong:Shandong University; 2014:53-54

[2] AiMu, Improvement and optimization of fiber optic temperature measurement system based on Raman scattering[D].Shandong:Shandong Maritime University; 2017:18-24

[3] HeWei, Research on cable temperature monitoring system based on distributed optical fiber and data processing[D].HuNan:Changsha University of Science and Technology;2011:26-30

[4] WangHaiFeng,Research on the method of improving the performance of distributed Raman fiber optic temperature sensing system[D].AnHui:Hefei Polytechnic University;2016:32-43 